\title{
MINERÍA DE AMIANTO: UNA APROXIMACIÓN A LOS RIESGOS TECNOLÓGICOS AMBIENTALES Y LA SALUD OCUPACIONAL
}

\author{
Antônio José Ribeiro Nunes ${ }^{1}$
}

\begin{abstract}
RESUMEN: Brasil es uno de los mayores productores mundiales de asbesto o asbesto, este constituye un mineral, cuyo fibrilo natural tiene sedosidad y propiedades físicas y químicas de gran valorpara la industria automotriz y de la construcción, debido a su resistencia mecánica y capacidad para soportar altas temperaturas. El amianto tiene un alto riesgo para la salud humana, con efectos devastadores. La minería en el Estado de São Paulo y Río de Janeiro fue prohibida, en vista de los problemas de los trabajadores afectados por la asbestosis, perodada su gran escala de uso en la industria nacional, la mayoría de las regiones brasileñas continúan con las actividades mineras. El propósito de este artículo es demostrar los riesgos tecnológicos y ambientales en las actividades productivas contemporáneas de la minería del asbesto. Su objetivo es demostrar los riesgos inminentes de las operaciones, los efectos sobre el cuerpo humano en los trabajadores con exposiciones prolongadas; enfermedades profesionales resultantes de la exposición continua al mineral. Los resultados muestran que el uso a gran escala en grandes sectores de producción determina su uso mediante la aplicación de medidas restrictivas y límites de exposición de los trabajadores. Aun así, este tema sigue siendo controvertido, considerando que São Paulo y Río de Janeiro abolieron eluso del asbesto ante los efectos negativos en la salud de los trabajadores. La Confederación Nacional de Trabajadores de la Industria - CNTI ha buscado alternativas en vista de la importancia del asbesto en la economía nacional, cuyas tendencias son la extracción controlada y la inspección con métodos de pruebas de contaminación ambiental y el uso obligatorio de EFI.
\end{abstract}

Palabras-clave: Minería. Asbesto. Asbestosis. Riesgos Tecnológicos Ambientales. Salud Ocupacional.

\section{INTRODUCCIÓN}

El uso de la carga de incrustaciones de asbesto en la industria brasileña ha generado muchas controversias y el intento de enmascarar problemas ambientales y ocupacionales relacionados con la minería del mineral que presenta "diferentes fibras de asbesto, tanto del grupo de los anfilolios (amosita, crocidolita, antofilita, actinolita y tremolita) como de las serpentinas (crisotilo)" (WUNSCH FILHO; NIEVE; MONCAU, 200I, p. I).

\footnotetext{
${ }^{1}$ Pós- graduado em auditoria, gestão e experiência ambiental. Graduado em Engenharia de Minas. E-mail: antonio.ribeiro@nm.gov.br
} 


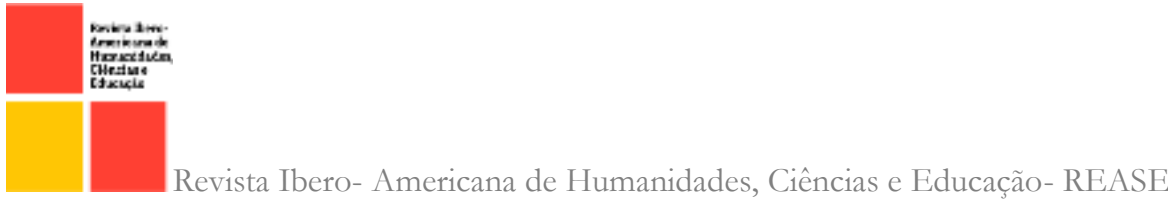

Actualmente existe el esfuerzo de adaptar y ampliar los Sistemas de Vigilancia e Información en Salud Ocupacional y Ambiental de los estados brasileños para la protección de los trabajadores que trabajan con la minería de asbesto. La vigilancia es compleja en el medida en que una acción de este tipo a menudo se lleva a cabo de forma clandestina. Aunque los estados de São Paulo y Río de Janeiro han determinado el fin de la explotación del asbesto, muchos municipios brasileños siguen manteniendo la explotación del mineral y los resultados en la salud de los trabajadores son enfermedades visibles y discapacidad física, muertes por neoplasias pulmonares por silicosis y otras enfermedades (BARCELLOS; QUITÉRIO, 2006).

El problema de la investigación apunta al siguiente análisis: ¿Cómo realizar la acción de vigilancia ambiental en el ámbito de las políticas de salud pública en las demandas relacionadas con el trabajo de los mineros de amianto?

Los supuestos del estudio indican que las alternativas más eficientes pueden ser el aumento regular de la supervisión en las regiones mineras, un mayor acceso a la atención de salud ocupacional, la ampliación de los procedimientos en los exámenes para la realización de diagnósticos y la regulación de la actividad con el fin de determinar medidas que eliminen alos ricos a través del uso de Equipos de Protección Personal - EPIs (CONFEDERAÇÃO NACIONAL DOS TRABALHADORES NAS INDÚSTRIAS - CNTI, 2013).

El propósito de este artículo es demostrar los riesgos tecnológicos y ambientales que surgenen las actividades productivas contemporáneas de la minería del asbesto. Su objetivo es demostrar los riesgos inminentes de las operaciones, los efectos sobre el cuerpo humano sobre los trabajadores que han estado expuestos durante períodos prolongados; enfermedades profesionales resultantes de la exposición continua al mineral.

La elección del tema en vista de la preocupación por la Seguridad y Salud ocupacional está justificada, considerando que el diagnóstico de enfermedades causadas por el mineral es complejo, además de contar con notificaciones raras de enfermedades profesionales en este campo de producción.

La relevancia del estudio es demostrar la importancia de la Seguridad y Salud en 
el Trabajo para la eficiencia de los procesos de estandarización de las estructuras de intervención en el entorno de trabajo de los mineros en vista de los intereses económicos en el mercado debido al valor del amianto y su gran uso en la industria.

En la actividad laboral existe una gran diversidad de riesgos laborales que requieren por parte de la salud pública y de las empresas diferentes responsabilidades en el ámbito de la Seguridad y Salud Laboral y la Higiene Ocupacional como ciencias que actúan directamente en la investigación y en la orientación de situaciones de riesgo ante los retos que plantea el sistema productivo.

\section{DESARROLLO}

I.I La extracción de amianto: un enfoque de los riesgos tecnológicos ambientales y la salud en el trabajo

Partiendo de la extracción de amianto y de los riesgos tecnológicos ambientales, la sociedad vive actualmente una vulnerabilidad constante expresada por el agravamiento de accidentes y enfermedades de carácter laboral que incluso las pautas de seguridad a veces no son suficientes ante los problemas tecnológicos ambientales generados por la producción.

Dentro de este enfoque de la vulnerabilidad, las directrices de Seguridad y Salud Ocupacional han buscado presentar propuestas integradoras con el objetivo de suplantar procedimientos fragmentarios, basados en fundamentos teóricos y metodológicos en técnicas de análisis de riesgos en un enfoque interdisciplinario y sistémico, que solo presentan resultados efectivos a través de la plena participación de los trabajadores en actividades de control de riesgos tecnológicos ambientales (CONFEDERAÇÃO NACIONAL DOS TRABALHADORES NAS INDÚSTRIAS - CNTI, 2013).

En este contexto de seguridad y salud ocupacional, se establece la definición de riesgos tecnológicos ambientales relacionados con efectos químico-radiactivos que producen grandes riesgos en las actividades productivas, centrándose en el análisis integral de "los peligros de la tecnología y sus dimensiones técnicas, cognitivas, sociales, culturales y filosóficas" (PORTO; FREITAS, 1997, p. 6o).

La responsabilidad de los organismos de protección de la salud de los trabajadores 
de dar el debido apoyo a la Política Nacional de Seguridad y Salud ocupacional que se cumpla efectivamente, desde el punto de vista de los riesgos tecnológicos ambientales que causan enfermedades ocupacionales en todos los sistemas de producción con el objetivo de determinar una interfaz entre la seguridad / higiene / salud y la dimensión ambiental en el trabajo a través de procedimientos estandarizados (MARANO, 2007).

En la minería del asbesto, los estudios epidemiológicos demuestran que el gran riesgo potencial es la enfermedad pleural obtenida por exposición, cuya alta prevalencia alcanza el $30 \%$ de las poblaciones mineras en estudios de hasta io años que demostraron la prevalencia de enfermedades como "el cáncer laríngeo y algunos tumores gastrointestinales también se relacionaron con el amianto en algunos estudios" (WUNSCH FILHO; NEVES; MONCAU, 2ooI, p. I).

Desde este punto de vista, las actividades laborales se estudian y analizan en cinco áreascon enfoques tecnológicos ambientales.

El amianto es un mineral que se encuentra fácilmente en la naturaleza y que por sus características peculiares como la flexibilidad y la resistencia a la tracción a las propiedades químicas, térmicas y eléctricas se utilizan comercialmente en diversos productos, como el cemento cuyas propiedades hacen del amianto un mineral utilizado como materia prima para la constitución de otros elementos, desde los vinculados a los componentes de la construcción civil. , como cemento, baldosas, caja de agua, así como en las industrias automotrices como lonas, frenos y recubrimientos de discos de embrague.

Según Martines e Grando (2008, p. or) debido a las propiedades del asbesto y entre ellas principalmente su resistencia, este mineral es utilizado en Brasil y en el mundo en la fabricación de diferentes productos comerciales. El autor también menciona que estáticamente aproximadamente el $85 \%$ son utilizados por las industrias del fibrocemento o fibrocemento, el Io\% en la industrialización de autopartes, el $3 \%$ en productos textiles y el2\% en la fabricación de productos químicos/plásticos.

Sin embargo, a pesar de estos beneficios químicos y físicos, el asbesto está formado por elementos que se puede demostrar que producen problemas de salud, como enfermedades pulmonares y cáncer. 
Para Medina (2008, p. 02) algunos estudios ya han demostrado que el amianto es un mineral, cuyos elementos químicos que lo componen tienen potencial cancerígeno. Estos elementosde potencial cancerígeno pueden detectarse en cualquier momento de la producción, transformación o incluso uso del mineral.

El autor también menciona que, según la Organización Mundial de la Salud (OMS), el crisotilo, un elemento asociado con el amianto, puede ser un factor desencadenante de numerosas patologías pulmonares, como la asbestosis, el cáncer de pulmón y el mesotelioma pleural yel peritono. Además, la investigación revela que no existe un límite seguro de exposición al riesgo cancerígeno que proporciona este componente químico.

En relación con asbestosis, según Martines y Grando (2008, p. 02) se considera que:

Es una enfermedad pulmonar relacionada con la inhalación prolongada de polvo que contiene una alta concentración de fibras de amianto. Las fibras se alojan en los alveolos pulmonares, y para defenderse, el cuerpo deposita sobre ellos una proteína similar a un "cemento" que cura el alveolo, evitando que se llene de aire.Este proceso, repitiéndose a lo largo de los años, puede hacer que el pulmón sea fibroso y sin elasticidad, con dificultades respiratorias.

Con respecto a otras patologías causadas por el contacto con amianto tenemos que en los casos de aparición de cáncer de pulmón, las mismas son más comunes cuando existe una asociación entre el tabaquismo y el carcinógeno. Por otra parte, según Martines e Grando (2008, p. 02) se considera que entre el inicio de la exposición a elementos de amianto hastael inicio del cuadro clínico del cáncer de pulmón, hay un período de tiempo de aproximadamente 20 años.

\subsection{Gestión de riesgos en entornos mineros}

La gestión de riesgos es una acción sistemática en organizaciones que desarrollan nuevos modelos de análisis de riesgos que buscan identificar, en la complejidad de los eventos tecnológicos ambientales en el trabajo, los factores que simultáneamente los desencadenan, las posibles condiciones de riesgo preexistentes en el trabajo (SOUZA; FONTES, 2008).

Veloso Neto (2013) es un devoto de la aplicación del sistema de gestión de la 
seguridad enlos mineros de amianto. La gestión de riesgos incorpora la necesidad de una inspección periódica de los lugares de trabajo con el fin de evaluar el uso de las EPIs por parte de los trabajadores, así como el tiempo de exposición basado en la reducción del tiempo deservicio.

En este contexto, corresponde a la empresa minera contratar a un especialista para gestionar la correcta eliminación del asbesto de manera que se observen las normas de seguridad de acuerdo con la NR - is (Anexo i2) que regula el uso en la forma de fabricación del producto, quedando prohibido el uso de algunos tipos de asbesto.

Normalización técnica, NBR 5643 (MBiogo) regula la fabricación de baldosas de amianto, NBRi3956-i que regula el proceso de exposición del trabajador a entornos donde existen riesgos de sílice mediante la determinación de las directrices de análisis mediante ensayosde nivel de sílice en el entorno minero.

Las demás normas técnicas del cuadro que figura a continuación se refieren a los análisis ambientales relativos a los métodos de ensayo de la contaminación ambiental y la concentración de productos químicos:

Cuadro i: Normas técnicas ambientales que pueden estar comprendidas en el uso del amianto por las empresas mineras.

\section{Normas Ambientais pela ABNT}

NBR

$8969 / 85$

Poluição do ar - Terminologia

NBR Dióxido de enxofre no ar ambiente - Determinação da concentração pelo método da

9546/86 pararrosanilina-Método de ensaio

NBR Material particulado em suspensão no ar ambiente - Determinação da concentração

$9547 / 86$ total pelo método do amostrador de grade volume - Método de ensaio

NBR Calibração de vazão, pelo método da bolha de sabão de bombas de baixa vazão

10562/88 utilizadas na avaliação de agentes químicos no ar - Método de ensaio

NBR

$12085 / 91$

Agentes químicos no ar - Coleta de aerodispersoides por filtração - Método de ensaio

Fuente: Veloso Neto (2013) 
Tavares (2004) analiza que los procesos de producción potencialmente peligrosos, como las acciones llevadas a cabo por los trabajadores en ambientes de minería de asbesto, enatmósferas potencialmente peligrosas debido a la sílice favorecen las probabilidades reales de riesgos frente a las fuerzas de producción que ponen en riesgo a los seres humanos.

Tabla 2: Gestión de riesgos

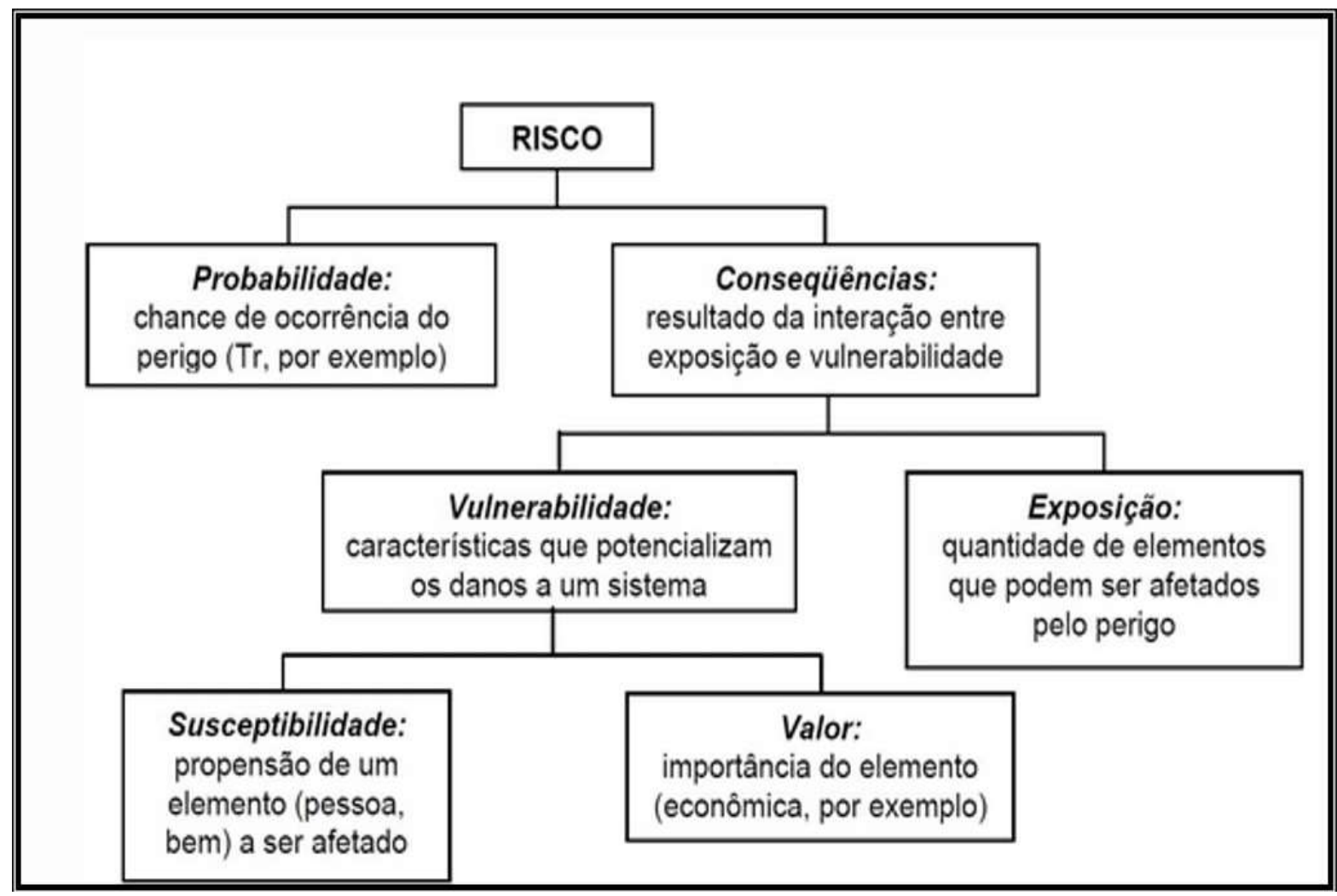

Fuente: Marano (2007)

Como se evidenció, la aplicación de los lineamientos de normas regulatorias favorece las instrucciones en el trabajo en materia de seguridad y salud, así como las medidas deprevención en los distintos ambientes de trabajo y actividades productivas que involucran el manejo del asbesto.

Los eventos peligrosos derivados de la exposición al amianto pueden hacer que el trabajador reaccione negativamente. La gravedad del evento es vista como una consecuencia del año generado que pueden lesiones, traumatismos, muerte, reducción de la capacidad de trabajo, etc. 
La gestión de riesgos en la minería del amianto implica la definición de riesgo que implica dos elementos importantes: la probabilidad de un evento de riesgo y la consecuencia peligrosa de este evento caracterizado por la prevalencia de enfermedades profesionales a medio y largo plazo.

Con respecto a este contexto legal y operativo del uso del asbesto, es de acuerdo con Martines y Brando (2008, p. 03) que existe el llamado Criterio de Salud Ambiental is lanzado por la Organización Mundial de la Salud. A través de este criterio, se orienta que en relación a las fibras biopersestentes del amianto se debe someter a un proceso de control rígido y permanente con el fin de minimizar los efectos tóxicos y cancerígenos.

En el caso de eventos de riesgo, estos se derivan de actividades producidas poniendo enriesgo la salud y la integridad física del trabajador. Estos eventos surgen del alto grado de riesgos de extracción de minerales de asbesto, asociados a situaciones de desinformación y falta de supervisión.

A pesar de todos los daños causados por el uso de asbesto por parte de las industrias, todavía no existen leyes específicas que prohíban la extracción, con la excepción de algunos estados brasileños como São Paulo y Rio de Janeiro, en otras situaciones de uso, la mayoría de los estados establecen criterios para controlar la explotación del asbesto, determinando eluso de EFI.

Se sabe que varias patologías son causadas por los elementos que forman el asbesto y que estas patologías no solo afectan a los trabajadores mineros, sino también a los que trabajanen industrias y fábricas, sino también a toda la población que reside cerca del entorno productor.

Por lo tanto, se considera que la lucha por el uso del seguro de amianto por parte de las industrias debe ser de todos, trabajadores y sociedad, que tienen derecho a vivir en unmedio ambiente sano y riesgo de daño.

\section{CONSIDERACIONES FINALES}

El estudio mostró las dificultades de prohibir el uso de asbesto en la industria brasileña. Su uso a gran escala en grandes sectores de producción determina su uso 
mediante la aplicación de medidas restrictivas y límites de exposición de los trabajadores. Aun así, este tema sigue siendo controvertido, considerando que São Paulo y Rio de Janeiro abolieron eluso del asbesto ante los efectos negativos en la salud de los trabajadores.

La Confederación Nacional de Trabajadores de la Industria - CNTI ha buscado alternativas en vista de la importancia del asbesto en la economía nacional, cuyas tendencias son la extracción e inspección controladas a través de métodos de prueba de contaminación ambiental y el uso obligatorio de EPIs.

Actualmente, la prevención de riesgos tecnológicos ambientales induce a la sociedad abuscar alternativas para evitar la vulnerabilidad de los trabajadores durante el proceso productivo, desde la gestión de riesgos que pueda reducir en la medida de lo posible el potencial nocivo de los riesgos de extracción y asbesto.

El mantenimiento de las actividades de extracción sin las debidas precauciones de las normas técnicas coloca al trabajador en gran vulnerabilidad con enfermedades profesionales que en cuanto al número de casos patológicos que han victimizado a los trabajadores, aunque actualmente no existen datos precisos, la investigación referida a las de 1980 y 2001 demuestra los resultados epidemiológicos sobre las enfermedades profesionales y sus graves riesgos.

La Constitución brasileña determinó el pleno derecho de los trabajadores a vivir en un ambiente de trabajo saludable y libre de riesgos para la salud, sin la proyección de ningúntipo de problemas tecnológicos ambientales generados por la producción.

Dentro de este enfoque de vulnerabilidad, se demostró la necesidad de gestión de riesgos, inspección periódica y creación de un equipo de profesionales para informar adecuadamentea los trabajadores de los riesgos.

\section{REFERENCIAS}

BARCELOS, Christovam; QUITÉRIO, Luiz Antônio Dias. Vigilância ambiental em saúde e sua implantação no Sistema único de Saúde. Revista de Saúde Pública, v. São Paulo, n. or, ed.4o, jun./fev. 2006. 
CONFEDERAÇÃO Nacional dos Trabalhadores nas Indústrias - CNTI. Acordo nacional para extração, beneficiamento e utilização segura e responsável do amianto crisotila 2013 a 2015 . Disponível em:

$\langle$ http://www.sinticomex.org.br/ckfinder/userfiles/files/ACT\%20CNTA\%202015.pdf〉. Acesso em: 5 mar. 2021.

MARANO, Vicente Pedro. Doenças ocupacionais. 2. ed. São Paulo: LTr, 2007.

MARTINES, Marco Utrera; BRANDO, Daniela. Amianto: ¿proibição, uso controlado ou imobilização? 2oo8. Disponível em:

$\langle$ http://ambientes.ambientebrasil.com.br/residuos/artigos/amianto\%3A_proibicao,_uso_contr olado_ou_imobilizacao\%3F.html> Acesso em: 6 mar. 2021.

MEDINA, Damares. O Amianto e as Restrições ao seu uso na Jurisprudência do Supremo Tribunal Federal. Revista Prática Jurídica, São Paulo, v. VII, n. 72, mar. de 2008.

SOUZA, Luis Cláudio Paiva de; FONTES, Carlos Eduardo Mazzuco. Qualidade de vida no trabalho: saúde emocional e gestão estratégica. São Paulo: EDICON, 2008.

TAVARES, José da Cunha. Noções de prevenção e controle de perdas em segurança dotrabalho. São Paulo: SENAC, 2004.

VELOSO NETO, Hernani. Construção social do risco e da segurança do trabalho em contexto organizacional. Rio de Janeiro: Civeri Publishing, 2013.

WUNSCH FILHO; V. NEVES, H.; MONCAU, J. E. Amianto no Brasil: conflitos científicos e econômicos. Revista Associação Médica Brasileira, São Paulo, v. 47, n. 3, jul./set. 200I 